\title{
Primitive but not simple
}

THE study of human embryonic haemoglobin, initiated principally by Huehns and his collaborators, starts from the demonstration that biosynthesis of a 'primitive' or $\varepsilon$ polypeptide chain precedes that of the $\gamma$ chains of human foetal haemoglobin (Hb-F, $\left.\alpha_{2} \gamma_{2}\right)$. At birth $\mathrm{Hb}-\mathrm{F}$ normally comprises some $80 \%$ of the total haemoglobin, the remainder being adult haemoglobin (Hb-A, $\alpha_{2} \beta_{2}$ ), as the biosynthesis of $\beta$ chains is switched on, and that of $\gamma$ chains starts to decline at 6 months' gestation. The $\varepsilon$ chain occurs as $\mathrm{Hb}$-Gower $1\left(\varepsilon_{4}\right)$ and $\mathrm{Hb}$ Gower $2\left(\alpha_{2} \varepsilon_{2}\right)$ and its formation has completely stopped at a gestational age of less than 3 months. There is also evidence that synthesis of the $\alpha$ chain is preceded very early in gestation by another type of chain, originally denoted as $x$ but now designated $\xi$. This chain is found in the haemoglobin species Hb-Portland 1 with composition $\xi_{2} \gamma_{2}$, believed to be present in early human embryos, and also found in stillborn infants with erythroblastosis foetalis caused by homozygous $\alpha$-thalassaemia 1 , where $\alpha$-chain synthesis is completely inhibited.

The practical problems associated with the study of human embryonic haemoglobins can be circumvented in animal studies, and Melderis, Steinheider and Ostertag, of the MaxPlanck Institute for Experimental Medicine, Göttingen, now report (see page 774 of this issue) evidence for a unique $\mathrm{x}$-type globin chain in early mammalian embryos (BALB/c mice and New Zealand white rabbits). The $x$ chains of these two species have closely related sequences, similar to that of the human $\xi$ chain of $\mathrm{Hb}$ Portland 1. Detailed analysis of the complex systems-three embryonic species in the mouse and six in the rabbit-leads to the conclusion that there are at least two different $\varepsilon$ chains, and that $\mathrm{x}$ - and $\alpha$-chain synthesis in both species is inversely correlated, hence the first chain takes the place of the second during early embryo- genesis. The $\mathrm{x}$ chain is now to be regarded as a $\alpha$-type chain, and there is more sequence similarity between $x$ chains of different species, than between the $\mathrm{x}$ and $\alpha$ chains of the same species. This indicates an early evolutionary divergence of $\mathrm{x}$ - and $\alpha$-chain genes and constitutes a further example of globin gene duplication during evolution; compare the findings of Schroeder, Huisman and colleagues on the duplicated ${ }^{\text {C }} \gamma$ and ${ }^{A} \gamma$ genes for the $\gamma$-chain of human Hb-F.

The Göttingen work confirms the indication from the earlier work on human embryonic haemoglobins that there is an $\alpha$-type $x$ (or $\xi$ ) chain synthesised in the nucleated erythroid cells derived from the yolk sac during early stages of mammalian embryogenesis, and preceding the synthesis of $\alpha$-chains proper. The presence of an $\varepsilon_{4}$ species in human but not in mice or rabbit embryos remains to be explained.

From a Correspondent

\section{Topology of crystal grains}

\section{from Robert W. Cahn}

IT is one of the paradoxes of the history of science that the rigorous quantitative treatment of the behaviour of large populations of molecules preceded by many years the similarly rigorous description of the individual molecules themselves; yet the kinetic theory of gases implies the extraction of orderly and predictable behaviour from myriads of random motions and collisions, whereas molecules are all identical. The resolution of the paradox lies in the fact that in the kinetic theory, molecules are treated virtually as independent, featureless particles, and therein lies the tractability of the whole approach. A million particles spell simplicity; one molecule spells complexity.

With crystals, the sequence is reversed. A large number of crystal structures had been accurately determined before the collective behaviour of populations of crystals began to be understood. The metallurgist recognises two distinct forms of such collective behaviour: first, there is 'Ostwald ripening', a poetically metaphorical term for the Matthew principle applied to a population of spherical crystallites of varying radii dispersed in a matrix; the larger ones grow at the expense of the smaller, essentially because of a radius- dependence of the solubility of the particles in the matrix. (Water droplets in a cloud behave in an essentially similar way.) The rigorous treatment of this very important metallurgical process is now well understood.

The second form of colleotive behaviour is grain growth. A piece of a pure metal consists of a population of crystal grains all sharing the same crystal structure but of different sizes, shapes and orientations. The grains grow from independent nuclei and as they impinge, grain boundaries are formed. The interfaces may be plane or curved, and bear no relation to the regular lattice arrangement of atoms: "There's no art to read the grain's construction in the face". Here, also, the Matthew principle operates: if a piece of metal is heated, the larger grains grow at the expense of the smaller through the migration of grain boundaries, and the average grain size progressively increases.

Grain growth has been studied micrographically for many years, both for its intrinsic interest and because of its practical influence on metal working, on the behaviour of nuclear fuel elements, in powder metallurgy and elsewhere. It has long been recognised that the mean grain diameter varies with time according to $\Delta D=k t^{n}$, where $n$ falls in the range $1 / 3-1 / 2$. The problem is to understand why. It is a conceptually more difficulit problem than the kinetic theory of gases, because crystal grains are not independent and separate particles, but connected polyhedra. When one grain changes shape and size, the neighbours of necessity change too. An assembly of grains is like a nuclear reactor: a change in neutron absorption in one small corner soon leads to a change in neutron flux throughout the reactor. Similarly, an instability at one corner of one grain quickly spreads through the population.

The key to an understanding of the problem is its topology. A population of polyhedral grains can be characterised by the number of grains, faces and vertices, and by the relations between these quantities. In particular, interest attaches to the mean number of faces per grain and the distribution of this number among the grain population. Topological principles were first applied, to assess the stability of a grain population, by C. S. Smith in 1952 . He recognised that grain growth stems from disequilibrium at edges where three grains meet and at four-grain corners. Unless three boundaries meeting at an edge are mutually inclined at $120^{\circ}$ the edge must move and the boundaries become curved. The curvature itself introduces instability; indeed, curvature and non-equilibrium edge and corner configurations are the fuels of grain growth. The nearest approach to equilibrium obtains in a population of fourteen-sided polyhedra of a particular shape.

In a brilliant paper Rhines, Craig and 